\title{
Clinical Study of Functional Outcome of External Fixator Device as a Primary Definitve Treatment of Open Tibia Fracture
}

\author{
Praveen Garg ${ }^{1}$, Rajvirsinh Sodha ${ }^{2}$, Fahad Bin Hamid ${ }^{3}$, T. Somashekarappa ${ }^{4}$ \\ ${ }^{1}$ Associate Professor, Department of Orthopaedics, RMCH, Bareilly, U.P., ${ }^{2} J u n i o r ~ R e s i d e n t$, Department of \\ Orthopaedics, RMCH, Bareilly, U.P., ${ }^{3}$ Assistant Professor, Department of Orthopaedics, RMCH, Bareilly, U.P., \\ ${ }^{4}$ Professor and Head, Department of Orthopaedics, RMCH, Bareilly, U.P.
}

\begin{abstract}
Background: Open fractures of leg are quite common in Road traffic accidents. The ideal protocol for open fractures of leg is initial thorough debridement, soft tissue coverage and intramedullary nailing or external fixator application. External fixator may be later converted to internal fixation with reamed or unreamed intramedullary nail or left as a definitive treatment.
\end{abstract}

Methodology: This prospective study was conducted at Rohilkhand Medical College And Hospital from January 2019 to January 2020 on patients presenting with open tibial fracture. Tubular External Fixator was used. Total 30 patients were enrolled in this study.

Results: In our study 30 patients were included in which 20 were male and 10 were females with mean age 32.2 years including children and elderly age group there was no age bar. Mean operative time was 60 minutes. Average time for union was 24 weeks.

Conclusion: External fixation as a primary definitive treatment for open tibial fracture is a cost effective procedure in developing country with minimal morbidity and mortality.

Keywords: Compound fracture, external fixator, Pin.

\section{Introduction}

Open fractures of leg are quite common in Road traffic accidents. The ideal protocol for open fractures of leg is initial thorough debridement, soft tissue coverage and intramedullary nailing or external fixator application. External fixator may be later converted to internal fixation with reamed or unreamed intramedullary nail or left as a definitive treatment. ${ }^{1}$

\footnotetext{
Corresponding Author:

Dr. Rajvirsinh Sodha

Junior Resident, Department of Orthopaedics, RMCH, Bareilly, U.P.

e-mail: sodharajvir@gmail.com

Mobile Number: 9925012456, 7457012288
}

The goals of open fracture management are prevention of infection, soft tissue coverage, achievement of bony union and restoration of function. Important principles involve antibiotic utilization, timing of initial surgical intervention, thorough debridement, type of wound closure and fixation of fracture after proper alignment. $^{2}$

The management depends on type of injury, contamination of wound, viability of skin and soft tissues and compartment syndrome. Though intramedullary interlocking nailing is used in open fractures of tibia. In reamed nail the infection rate has been reported high. A high incidence of infection has been reported in delayed intramedullary nailing ${ }^{3}$. There is controversy in literature regarding the best way of management of type II and type III open tibia fractures ${ }^{4}$. Ex fix as a definitive procedure reduces the cost of one more surgery. 
Aim and Objectives: To assess the functional outcome of external fixator device as a primary definitive treatment for open tibia fracture.

\section{Materials and Method}

The study was done at Rohilkhand Medical College And Hospital from January 2019 to

January 2020.

Total 30 patients were included, and all patients treated with external fixator as definitive treatment after thorough debridement and proper reduction and alignment of fracture.

\section{Inclusion Criteria:}

Open fractures of both bones of leg type II, type III A and B (Gustilo Anderson)

\section{Exclusion Criteria:}

Fractures with bone defects

Intra-articular fractures.

Surgical technique: The patients with compound fracture injury taken up for surgery on OT table, proper scrubbing of limb and draping done, prophylactic antibiotics was given.

Thorough wound debridement and wound wash with normal saline was done

Proximal and Distal pins applied on arterio-medial aspect of tibia

Anatomical reduction and alignment were done and connecting rods was applied

If the tissue and skin viability is good, a primary closure was done without any tension.

If necessary release incision was given for primary closure of wound.

Wound swab was taken for culture and sensitivity before closure.

Soft tissue coverage by flap or skin grafting was done within two weeks in patients in whom primary closure was not done.

Depending on the culture sensitivity of the organism appropriate antibiotics were continued for ten days.

The pins were cleaned and dressed with beta dine on alternate days.

Follow Up: Each patient was evaluated clinically and radiographically at three weeks, six weeks, 3 months, then after every four week to assess the union of the fracture.

Pin tract infections were treated by dressing, appropriate antibiotics.

Fracture healing was assessed by standard radiographic projections and union defined as dense callus bringing at least three cortices.

Partial weight bearing was allowed at 6 weeks and full weight bearing at around 3 months.

The external fixator was removed when there is union of the fracture. A pop slab was given for two weeks for the healing of pin tracks.

The patients were assessed for healing of soft tissues, fracture union, function of knee and ankle.

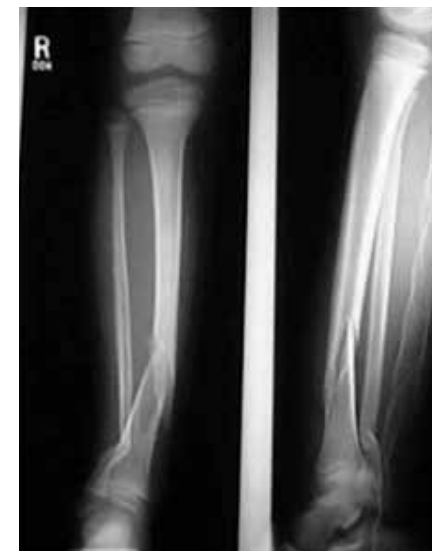

(a)

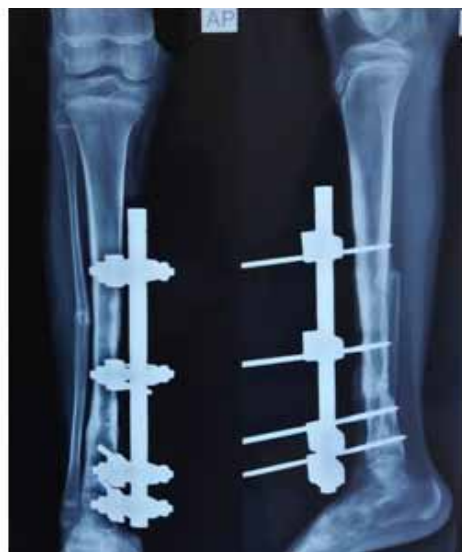

(b)

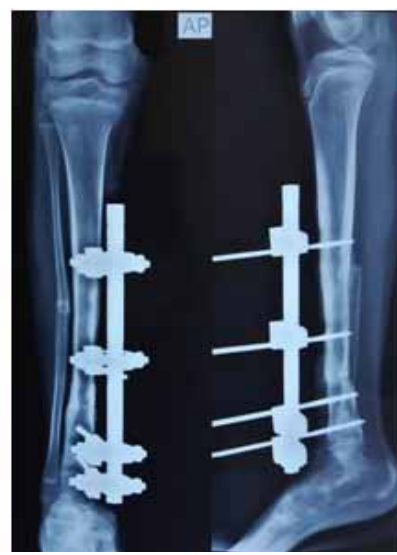

(c)

Fig (a) Pre-operative X ray (b) 6 weeks post-operative (c) 12 weeks post-operative 


\section{Result}

In our study 30 patients were included in which 20 were male and 10 were females with mean age of 32.2 years. Mean operative time was 60 minutes. Average time for union was 24 weeks.

The level of fracture was at middle $1 / 3$ in 22 and lower $1 / 3$ in 5 cases and upper $1 / 3$ in 3 cases. The grade of open fracture was type II in 20, type III A in 6 and type III B in 4 patients.

Primary closure was done in 22 patients while secondary closure was done in 5 patients and flap coverage was done in 3 patients.Complication like loss of reduction occurred in 3 patients, pin tract infection was occurred in 6 patients, pin loosening in 1 patient.

The fracture union by $10-12$ weeks in 2 patients, by 12-16 weeks in 6 patients, 16-20 weeks in 14 patients, and 20-24 weeks in 8 patients.

Malunion was defined as varus or valgus malalignment of 5 degree or more, anterior or posterior angulation of 10 degree or more, shortening of $1 \mathrm{~cm}$ or more or rotational malalignment of 10 degree or more as compared with the contralateral leg.

In our study there were 3 patients had nonunion and 3 patients had delayed union. Pin tract infection was major complication of the external fixator. In our study 6 patients had pin tract infection. This was managed by pin tract dressing and antibiotics.

No patient had osteomyelitis like complication. There was no restriction of range of motion of knee and ankle joint.

\section{Discussion}

Open tibial fractures are associated with infection, soft tissue injury, malunion andnon-union thus leads to greater challenge ${ }^{4,5,6}$. These cases should be managed as early as possible to minimize complications. Intramedullary nailing is not possible due to contaminated wound thus external fixator is method of choice. External fixator has advantages of low blood loss and proper wound care.

1. Comparison of Mean Age: Mean age in present study was 32.3 years compared to 25.5 years in Irfan $\mathrm{S}$ et al ${ }^{4}$ study which suggest older age group involved in present study while in A. Velazco et al ${ }^{5}$ it was 40.2 Years.
Age group 30-50 are productive age group who have high risk of injury.

2. Gender Comparison: Male predominance was found in both studies which may be attributed to higher risk of fracture in this population. In present study male population was 20 and female population was 10 as compared to A. Velazco et $\mathrm{al}^{5}$ and Ravi Kumar et $\mathrm{al}^{6}$ has more male population.

3. Comparison of Level of Fracture: Most common was Middle $1 / 3^{\text {rd }}$ fracture followed by lower and upper $1 / 3^{\text {rd }}$ in present study and Ravi Kumar et al ${ }^{6}$ as in our study middle fracture was 22 and in Ravi Kumar et al ${ }^{6}$ was 26 patients. It may be attributed to mechanics of tibial fracture which suggests middle $1 / 3$ having high risk. ${ }^{5,6}$

\section{Comparison of Fracture Union:}

Table 1: Comparison of Fracture Union:

\begin{tabular}{|c|c|c|}
\hline Weeks & Present Study & Irfan S et al $^{\mathbf{4}}$ \\
\hline $10-12$ & 2 & 1 \\
\hline $12-16$ & 6 & 2 \\
\hline $16-20$ & 14 & 10 \\
\hline $20-24$ & 8 & 2 \\
\hline$>25$ & 0 & 5 \\
\hline
\end{tabular}

In most of cases healing was found between 16-20 weeks in both studies. In Irfan S et al study 5 patients took $>25$ weeks' time to heal.

\section{Comparison of complications:}

Table 2: Comparison of complications

\begin{tabular}{|l|c|c|}
\hline Complications & Present Study & Ravi Kumar et al \\
\hline Loss of reduction & 3 & 3 \\
\hline Pin tract infection & 6 & 8 \\
\hline Pin loosening & 1 & 1 \\
\hline Delayed Union & 3 & 2 \\
\hline Non-Union & 3 & 3 \\
\hline
\end{tabular}

Pin tract infection was most common complications in both studies which may be due to local site wound contaminations which can be managed with antibiotics and dressing. Loss of reduction occurred in 3 patient which was managed conservatively. Pin loosening was found in 1 patient in each study. Delayed union in 3 patients in present study while Ravi Kumar et al reported 2 patients. There were non-union in 3 patients in present study while Ravi Kumar et al reported 3 patients. 


\section{Conclusion}

External fixation as a primary definitive treatment for open tibial fracture is a cost effective procedure in developing country with minimal morbidity and mortality.

Ethical Clearance: Taken from Institutional Ethical Committee Rohilkhand Medical College And Hospital

Source of Funding: Self

Conflict of Interest: Nil

\section{References:}

1. Giannoudis PV, Papakostidis C, Roberts C. A review of management of open fracture of tibia and femur. JBJS 2006; 3(88B):281-289

2. Robert W Bucholz, James D Heckman, Charles M, Court Brown. Rock wood and Green fracture in adults, $6^{\text {th }}$ Edition, 2079. 2014.

3. Michail Beltrias, Olga Sarridou, John Kovam's. External fixation on a primary and treatment for tibial diaphyseal fracture strategies trauma limb reconstruction. 2009; 4(2):81-87

4. Dr. SK Irfan Ali, Dr. Sujai S, Dr. HK MdJunied, Dr. Chethan M H, Dr. Ganesh H and Dr. MK Siddalinga Swamy. Evaluation of the functional outcome in open tibial fractures managed with an Ilizarov fixator as a primary and definitive treatment modality. International Journal of Orthopaedics Sciences 2017; 3(2): 436-440

5. A. Velazco, L. L. Fleming. Open Fractures of the Tibia Treated by the Hoffmann External Fixator. Clinical Orthopedics and related research.; 1982:(183):125-132.

6. Dr. Ravi Kumar, Dr. Sujai S, Dr. Chethan, Dr. HC Nanjundappa, Dr. Siddalinga Swamy. Results of open fractures of tibia treated by externalfixator as primary and definitive procedure. International Journal of Orthopaedics Sciences 2017; 3(1): 179181. 